\title{
Detection of Antibiotic Resistance Patterns in Staphylococcus aureus Strains Isolated from Patients Referring to Shahid Sadoughi Hospital in Yazd During 2017-2018
}

Jamshid Ayatollahi ${ }^{1}$, Motahara Salimi ${ }^{2}$, Zohreh Akhoundi Meybodi ${ }^{3}$, Tahereh falah ${ }^{4}$, Mahdie Hamidfar $^{1 *}$, Seyed Hossein Shahcheraghi ${ }^{1}$

${ }^{1}$ Infectious Diseases Research Center, Shahid Sadoughi Hospital, Shahid Sadoughi University of Medical Sciences, Yazd, Iran.

${ }^{2}$ Medical student, Shahid Sadoughi University of Medical Sciences, Yazd, Iran.

${ }^{3}$ Infectionlogist, Fellowship of prevention and control of nosocomial infections, Shahid Sadoughi Hospital, Shahid Sadoughi University of Medical Sciences, Yazd, Iran.

${ }^{4}$ Expert of statistics, RDC center, Shahid Sadoughi Hospital, Shahid Sadoughi University of Medical Sciences, Yazd, Iran.

*Corresponding Author: Mahdie Hamidfar, Infectious Diseases Research Center, Shahid Sadoughi Hospital, Shahid Sadoughi University of Medical Sciences, Yazd, Iran.

\section{Abstract}

Introduction: Staphylococcus aureus is one of the most important pathogens causing various forms of infection and is a major nosocomial infection agent. In recent years, due to the inappropriate use of antibiotics, drug resistance among microorganisms, including Staphylococcus aureus has increased. This study was conducted to determine the antibiotic resistance pattern of staphylococcus aureus spices isolated from patients referring to Shahid Sadoughi Hospital in Yazd during 2017-2018.

Materials and Methods: For this descriptive-analytic study, all positive cultures of Staphylococcus aureus in patients referred to Shahid Sadoughi Hospital in Yazd during 2017-2018 were evaluated. The samples included blood culture, urine culture, wound discharge culture, throat culture, peritoneal culture, pleural culture, synovial culture, CSF culture, bronchial lavage culture. Antibiotic resistance patterns of the samples against 13 different antibiotics were determined by the standard method of Disc Diffusion and data analyzed by SPSS 22 software.

Results: The highestantibiotics resistance was seen againstPenicillin (92.4\%), Tetracycline (52\%), Erythromycin (50\%), Cefoxitin (40.5\%), Doxycycline (36.4\%), Ciprofloxacin (34.3\%), Clindamycin (33.6\%), Cotrimaxazole (25.3\%) and the lowest resistance levels were against gentamycin (18.6\%).

Conclusion: The result of this study similar to the result of other studies shows the high prevalence of drug resistance among Staphylococcus aureus strains that is caused by inappropriate administration of antibiotics. The high sensitivity of Linezolid and Vancomycin shows important role of these antibiotics in treatment of resistance cases.

Keywords: Staphylococcus aureus; Antibiotics; Drug resistance

\section{INTRODUCTION}

Staphylococcus aureus is one of the most important and abundant causes of nosocomial infections worldwide [1]. Due to the prevalence of infectious diseases in the world, evaluation of antibiotic resistance is very important $[1,2]$. The investigation of drug resistance pattern has an important role in the proper selection of antibiotics, infection control and also treatment costs reduction. Currently, the spread of methicillin- 
Detection of Antibiotic Resistance Patterns in Staphylococcus aureus Strains Isolated from Patients Referring to Shahid Sadoughi Hospital in Yazd During 2017-2018

resistant staphylococcus aureus (MRSA) strains has become one of the main therapeutic challenges that is producing soft tissue and skin infections in the United States and is primarily responsible for the development of postoperative infections [3]. The multidrug resistant bacteria are one of the main problems in many countries. The most antibiotics that bacteria have a resistance towards them include beta-lactams such as penicillin and methicillin, and cephalosporin [2]. Recent studies have estimated that $8-12 \%$ of hospitalized patients suffer from the side effects of this infections. Identifying and treating patients can decrease the incidence of MRSA [4]. The researches have shown that infections caused by antibiotic-resistant organisms are associated with higher mortality than infections caused by susceptible strains [4, 5]. For example, a study in Canada found that the mortality of methicillin-resistant Staphylococcus aureus strains differed significantly from that of methicillin non-resistant Staphylococcus aureus in surgical patients [6]. Methicillin-resistant hospital stay. Methicillin-resistant Staphylococcus aureus is also more resistant to erythromycin, gentamicin, cotrimoxazole, and ciprofloxacin [6]. However, the pattern of drug resistance of microorganisms in hospital plays an important role in the proper selection of antibiotics, infection control and also costs reduction [7]. Awareness of bacterial resistance can also be a factor in preventing the overuse of antibiotics as an important factor in the development of antibiotic resistance [8]. Due to the prevalence of Staphylococcus aureus and the increase in resistance of this bacterium in recent years, this study was performed to determine the pattern of antibiotic resistance of Staphylococcus aureus isolated from patients in the laboratory of Shahid Sadoughi Hospital in Yazd.

\section{METHOD}

This descriptive and cross-sectional study was performed on positive culture samples for Staphylococcus aureus isolated from patients. The laboratory of Shahid Sadoughi Hospital in Yazd, Iran was conducted from the beginning of October 2017 to the beginning of October 2018. Sampling was performed by census and included all positive cultures for Staphylococcus aureus in the patients referred to the laboratory of Shahid Sadoughi Hospital in Yazd. Staphylococcus aureus increases the length of patients

Staphylococcus aureus positive samples were extracted by referring to the hospital information system. Then, information was collected using a questionnaire that was done based on surveys. This information included age, sex, type of admission, date of admission, type of sample and antibiogram results. In this study, the susceptibility and resistance of Staphylococcus aureus were evaluated by disk diffusion sensitivity test method. The antibiotics were including vancomycin, clindamycin, cotrimaxazole, ciprofloxacin, rifampin, tetracycline, erythromycin, cefoxitin, doxycycline, nitrofurantoin, penicillin, linezolid, and gentamicin. Sample types were including blood, urine, wound secretions, pharyngeal secretions, pleural fluid, peritoneal fluid, synovial fluid, cerebrospinal fluid, and bronchial lavage.

\section{RESULTS}

During 12 months of study, 241 culture samples were positive for Staphylococcus aureus. The average age of individuals was 44 years, the minimum age was one day and the maximum was 94 years. There were 134 samples (55.6\%) male and 107 samples (44.4\%) female. $90.9 \%$ of the samples were related to hospitalized patients and $9.1 \%$ were related to outpatients. Out of 241 samples, 100 samples (41.5\%) were related to wound secretions, 78 samples (32.4\%) blood, 35 samples (14.5\%) urine, 10 Sample (4.1\%) synovial fluid, 7 samples $(2.9 \%)$ pleural fluid, 5 samples (2.9\%) peritoneal fluid, 3 samples $(1.2 \%)$ secretions throat, 2 samples $(0.8 \%)$ bronchial lavage, and finally 1 sample $(0.4 \%)$ cerebrospinal fluid (Table 1).

Table1. The sample types related to patients

\begin{tabular}{|l|l|l|}
\hline Sample type & Abundance & Percentage \\
\hline Wound discharge & 100 & 41.5 \\
\hline Blood & 78 & 32.4 \\
\hline Urine & 35 & 14.5 \\
\hline Synovial fluid & 10 & 4.1 \\
\hline Pleural fluid & 7 & 2.9 \\
\hline Peritoneal fluid & 5 & 2.1 \\
\hline Throat discharge & 3 & 1.2 \\
\hline Bronchial lavage & 2 & 0.8 \\
\hline Cerebrospinal fluid & 1 & 0.4 \\
\hline total & 241 & 100 \\
\hline
\end{tabular}


Detection of Antibiotic Resistance Patterns in Staphylococcus aureus Strains Isolated from Patients Referring to Shahid Sadoughi Hospital in Yazd During 2017-2018

The pattern of the resistance of Staphylococcus aureus to 13 types of antibiotics was investigated. Out of 63 samples, $62(4.98 \%)$ were sensitive to linezolid and $1(6.1 \%)$ was resistant. Out of 227 samples, 216 $(2.95 \%)$ were sensitive to vancomycin, $4(8.1 \%)$ were semi-sensitive and 7 (1.3\%) were resistant. Out of 222 samples, 209 (1.94\%) were sensitive to nitrofurantoin, 2 (9.0\%) were semi-sensitive and 11 (5.5\%) were resistant. Out of 215 samples, 185 cases (86\%) were sensitive to rifampin and 30 cases $(14 \%)$ were resistant. Out of 215 samples, 174 cases (80.9\%) were sensitive to gentamicin, 1 case $(0.5 \%)$ was semi-sensitive, and 40 cases $(6.18 \%)$ were resistant. Out of 198 samples, $143(2.72 \%)$ sensitive, 5 (2.5\%) semi-sensitive and $50(3.25 \%)$ were resistant to cotrimaxazole. Out of 232 samples, 153 cases $(9.65 \%)$ were sensitive to clindamycin, 1 case $(0.4 \%)$ was semi-sensitive, and 78 cases (6.33\%) were resistant. Out of 137 samples, 87 cases $(63.5 \%)$ were sensitive to ciprofloxacin, 3 cases (2.2\%) were semi-sensitive, and 47 cases $(3.34 \%)$ were resistant. Out of 228 samples, 138 (60.5\%) sensitive, 7 (1.3\%) semi-sensitive, and 83 (4.36\%) were resistant to doxycycline. Out of 220 samples, $138(2.58 \%)$ were sensitive to sefoxetine, $3(4.1 \%)$ were semi-sensitive, and $89(40.5 \%)$ were resistant. Out of 234 samples, 115 (49.1\%) were sensitive to azithromycin, 2 (9.0\%) were semi-sensitive, and 117 (50\%) were resistant. Out of 173 samples, 83 (48\%) were sensitive to tetracycline and 90 (52\%) were resistant. Out of 224 samples, 17 (6.7\%) were sensitive to penicillin and 207 (4.92\%) were resistant. Among the antibiotics, the highest sensitivity was related to linezolid with $98.4 \%$, Vancomycin with $95.2 \%$ and nitrofurantoin (94.1\%) and the lowest sensitivity was related to penicillin (7.6\%) (Figure 1).

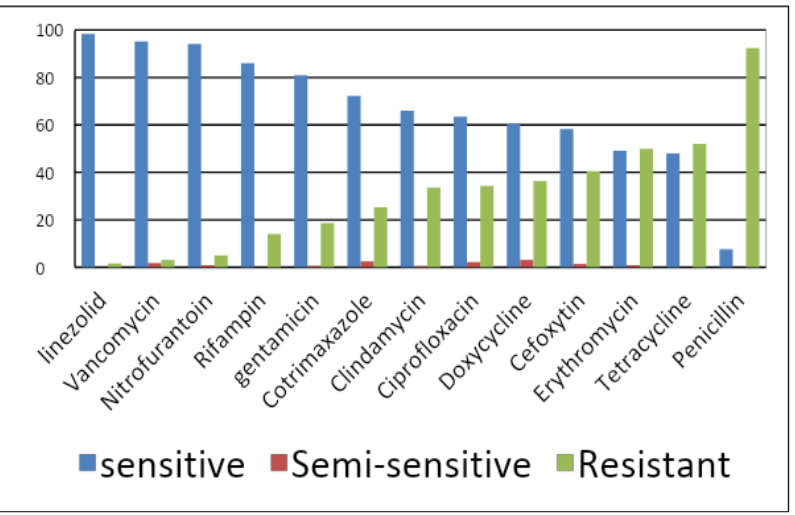

Figure1. The pattern of the resistance of Staphylococcus aureus to the antibiotics
The highest sensitivity in hospitalized cases was to linezolid (98.1\%), vancomycin (96.6\%), nitrofurantoin $(94.5 \%)$, rifampin (86\%), gentamicin (80.3\%) and the highest resistance was seen in penicillin (93.1\%). In outpatients, the highest sensitivity was to linezolid antibiotic with 100\%, nitrofurantoin (90.9\%), rifampin (86.4\%), gentamicin (86.4\%) and vancomycin $(81.8 \%)$ and the highest resistance was to penicillin (85\%).There was no significant difference between inpatients and outpatients in any of the antibiotics. In the samples of wound secretions, the highest sensitivity to antibiotics was related to linezolid (100\%), vancomycin (95.8\%), nitrofurantoin (93.3\%), and rifampin (85.4\%). Also, $96.7 \%$ of the samples were resistant to penicillin. The highest susceptibility of blood samples to antibiotics was $100 \%$ and related to linezolid and following vancomycin (97.1\%), nitrofurantoin (94.6\%), rifampin (86.1\%) and highest resistance (89.2\%) was to penicillin. In urine samples, the highest sensitivity was to linezolid (92.9\%), and then nitrofurantoin (90.6\%), vancomycin (87.9\%), rifampin and gentamicin (87.5\%). The lowest sensitivity was seen to penicillin (11.8\%).

$100 \%$ of the synovial fluid samples were sensitive to linezolid, vancomycin and nitrofurantoin and also resistant to penicillin. The sensitivity of synovial fluid samples to rifampin, gentamicin cotrimaxazole, clindamycin, ciprofloxacin and doxycycline was above $80 \%$. Pleural fluid samples were $100 \%$ sensitive to the linezolid, nitrofurantoin, and vancomycin. Hypersensitivity to ciprofloxacin, doxycycline, and rifampin was above $80 \%$. $83.3 \%$ of the samples were resistant to penicillin. The sensitivity of peritoneal fluid samples to the nitrolurantoin, gentamicin, clindamycin, ciprofloxacin, doxycycline, cefoxetine, erythromycin, and tetracycline was 100\%. The lowest sensitivity was seen to penicillin (20\%).Throat secretion samples were $100 \%$ sensitive to linezolid, vancomycin, nitrofurantoin and rifampin and 100\% resistant to clindamycin, ciprofloxacin, doxycycline, cefoxetine, erythromycin, tetracycline and penicillin. All bronchial lavage specimens were sensitive to the vancomycin, nitrofurantoin, rifampin, gentamicin, cotrimaxazole, clindamycin, doxycycline, cefoxetine, erythromycin, and tetracycline but they were resistant to penicillin. The samples of cerebrospinal fluid studied were sensitive to vancomycin, nitrofurantoin, gentamicin, clindamycin, ciprofloxacin, doxycycline, 
Detection of Antibiotic Resistance Patterns in Staphylococcus aureus Strains Isolated from Patients Referring to Shahid Sadoughi Hospital in Yazd During 2017-2018

cefoxetine, erythromycin and tetracycline and resistant to penicillin. There was a significant difference between the sensitivity of different samples to two antibiotics of cefoxetine $(\mathrm{p}=0.023)$ and erythromycin $(\mathrm{p}=0.008)$. In men, the highest sensitivity was to linezolid $(97.1 \%)$, vancomycin $(95 \%)$, nitrofurantoin (94.5\%), rifampin $(85.7 \%)$, gentamicin $(81.8 \%)$ and the lowest sensitivity to penicillin (4\%), tetracycline (45.2\%) and erythromycin (52.9\%). In women, the highest sensitivity to antibiotics was related to linezolid (100\%), vancomycin (96\%), nitrofurantoin (93.1\%), rifampin $(86.5 \%)$, gentamicin $(79.8 \%)$ and the lowest sensitivity was to penicillin with $12 \%$. The significant difference was not observed between men and women in any of the antibiotics. Also, a significant difference was observed between age groups about ciprofloxacin, clindamycin, doxycycline and rifampin, which could be due to the small number of samples in some age groups.

\section{DISCUSSION}

In recent years, due to the inappropriate use of antibiotics, the resistance of various bacteria, including Staphylococcus aureus, has increased. Therefore, determining the pattern of antibiotic resistance to select the appropriate treatment of infections caused by this bacterium seems necessary. This study was performed to investigate the pattern of antibiotic resistance of Staphylococcus aureus isolated from patients in the laboratory of Shahid Sadoughi Hospital in Yazd in 2017-2018. The findings of a recent study on Staphylococcus aureus isolated from blood, urine, wound secretions, pharyngeal secretions, pleural fluid, peritoneal fluid, joint fluid, cerebrospinal fluid, bronchial lavage showed that the highest antibiotic susceptibilities were related to linzolid (98.4\%), vancomycin (95.2\%), nitrofurantoin (94.1\%), rifampin (80.9), gentamicin (72.2), clindamycin (65.9), ciprofloxacin (63.5), and doxycycline (48.5), respectively. And also, the lowest sensitivity was to penicillin (6.7\%). In the present study, the resistance to beta-lactams was $92.4 \%$ for penicillin and $40.5 \%$ for cefoxitin. Resistance to erythromycin was $50 \%$, clotrimazole $25 \%$, gentamicin $18.6 \%$, nitrofurantoin $5 \%$. Resistance to ciprofloxacin increased by $34.3 \%$ and vancomycin $3.1 \%$. In a study, the prevalence of the mecA methicillin resistance gene was investigated and antibiotic resistance patterns were determined in S.aureus Isolated strains from clinical specimens in Tabriz, Iran [9]. The phenotypic evaluation of the antibiotic resistance pattern of methicillin-resistant S.aureus showed that the highest resistances were to penicillin (100\%) and Co-Amoxiclav (91.66\%). Molecular examination revealed the presence of $68 \%$ the mecA gene in isolates [9].

A research in Colombia was performed on 976 positive Staphylococcus aureus culture samples. $49.6 \%$ of the samples were resistant to methicillin. Of these samples, simultaneous resistance to tetracycline $42.6 \%$, erythromycin $31 \%$, clindamycin $29.4 \%$, ciprofloxacin $25.1 \%$, gentamicin $25 \%$, cotrimaxazole $11.8 \%$, vancomycin $2.8 \%$ was observed. Also in methicillin-sensitive samples, tetracycline resistance was relatively high (31.6\%). All samples were linezolid sensitive [10].

The frequency and pattern of antibiotic resistance strains at Mashhadad Imam Reza Hospital were determined in another study. In this research, 925 strains of S.aureus (6.12\%) were identified from a total of 7335 strains isolated from patients hospitalized in this hospital. The MRSA incidence was 382 (7.41\%) cases. The bulk of MRSA was isolated from cultured sampling of blood and wounds. Internal, Burn and Cardiology Wards had the most cases of MRSA [11].

A study was performed to determine the resistance pattern of the most common bacterial strains of blood and urinary tract in the hospital during two years. The highest resistance was related to penicillin $(91.1 \%)$ followed by ampicillin (75.6\%), and the lowest rate was against vancomycin (4.4\%) [12].

Therefore, the present study showed that the highest antibiotic susceptibility to antibiotics used in the treatment of infections caused by Staphylococcus aureus are related to linezolid, vancomycin, rifampin, gentamicin, cotrimaxazole, respectively. Clindamycin, ciprofloxacin, doxycycline, cefoxitin and the highest resistance are related to penicillin, tetracycline and erythromycin, respectively. Hypersensitivity to over $90 \%$ of linezolid and vancomycin is important in the treatment of infections caused by resistant strains, and it is hoped that this pattern will be maintained with the proper use of these antibiotics. High resistance of $92.4 \%$ penicillin can be due to improper use of this antibiotic. 
Detection of Antibiotic Resistance Patterns in Staphylococcus aureus Strains Isolated from Patients Referring to Shahid Sadoughi Hospital in Yazd During 2017-2018

\section{REFERENCES}

[1] Harris LG, Foster S, Richards RG (2002) An introduction to Staphylococcus aureus, and techniques for identifying and quantifying S. aureus adhesins in relation to adhesion to biomaterials: review. Eur Cell Mater 4(3):10020.

[2] Dibah S, Arzanlou M, Jannati E, Shapouri R (2014) Prevalence and antimicrobial resistance pattern of methicillin resistant Staphylococcus aureus (MRSA) strains isolated from clinical specimens in Ardabil, Iran. Iranian journal of microbiology 6(3):163.

[3] Morell EA, Balkin DM (2010) Methicillin-resistant Staphylococcus aureus: a pervasive pathogen highlights the need for new antimicrobial development. The Yale journal of biology and medicine 83(4):223-33.

[4] Köck R, Becker K, Cookson B, van Gemert-Pijnen J, Harbarth S, Kluytmans J, et al (2010) Methicillinresistant Staphylococcus aureus (MRSA): burden of disease and control challenges in Europe. Eurosurveillance 15(41):19688.

[5] Dehghani A, Gholipour A, Nazari-Vanani R, Heibati F (2015) Molecular investigation of methicillin-resistantStaphylococcus epidermidis and Staphylococcus saprophyticus isolated in Shahrekord training hospitals. Journal of Shahrekord Uuniversity of Medical Sciences 17.

[6] Kluytmans J, Struelens M (2009) Meticillin resistant Staphylococcus aureus in the hospital. BMJ 338:b364.
[7] Abbasi-Montazeri E, Khosravi AD, Feizabadi MM, Goodarzi H, Khoramrooz SS, Mirzaii M, et al (2013) The prevalence of methicillin resistant Staphylococcus aureus (MRSA) isolates with high-level mupirocin resistance from patients and personnel in a burn center. Burns 39(4):650-4.

[8] Gardete S, Tomasz A (2014) Mechanisms of vancomycin resistance in Staphylococcus aureus. The Journal of clinical investigation 124(7):283640.

[9] Jafari-Sales A, Jafari B (2019) Evaluation of the Prevalence of mec A Gene in Staphylococcus aureus Strains Isolated from Clinical Specimens of Hospitals and Treatment Centers. Pajouhan Scientific Journal 17(3):41-7.

[10] Pérez N, Pavas N, Rodríguez EI (2010) Antimicrobial resistance of Staphylococus aureus at a hospital from the Colombian Orinoquia. Infection 14(3):167-73.

[11] Tabaei S, Kouhi Noghondar M, Mohammadzadeh M, Ataei L, Amel Jamehdar S (2016) Pattern of antibiotic resistance in methicillin-resistant Staphylococcus aureus (MRSA) strains isolated from clinical specimens: Imam Reza hospital in Mashhad. medical journal of mashhad university of medical sciences 59(2):64-70.

[12] Ghadiri H, Vaez H, Khosravi S, Soleymani E (2012) The antibiotic resistance profiles of bacterial strains isolated from patients with hospital-acquired bloodstream and urinary tract infections. Crit Care Res Pract 2012.

Citation: Jamshid Ayatollahi, et al. Detection of Antibiotic Resistance Patterns in Staphylococcus aureus Strains Isolated from Patients Referring to Shahid Sadoughi Hospital in Yazd During 2017-2018. Open Access Journal of Internal Medicine. 2020; 3(1): 27-31.

Copyright: (c) 2020 Jamshid Ayatollahi, et al. This is an open access article distributed under the Creative Commons Attribution License, which permits unrestricted use, distribution, and reproduction in any medium, provided the original work is properly cited. 